\title{
Técnica de cópia de próteses totais para pacientes idosos: relato de caso
}

\author{
Marcella Silva de PAULA®'; Angélica MAGALHÃES NETA2; Talitha Maria \\ Cabral OLIVEIRA ${ }^{3}$; Cláudio Rodrigues LELES ${ }^{4}$; Gerald MCKENNA ${ }^{5}$
}

1 - Doutoranda em Clínica Odontológica, Faculdade de Odontologia, Universidade Federal de Goiás, Goiânia, GO, Brasil; 2 - Aluna de graduação em Odontologia, Faculdade de Odontologia, Universidade Federal de Goiás, Goiânia, GO, Brasil; 3 - Mestranda em Clínica Odontológica, Faculdade de Odontologia, Universidade Federal de Goiás, Goiânia, GO, Brasil; 4 - Professor Titular de Prótese Dentária, Faculdade de Odontologia, Universidade Federal de Goiás, Goiânia, GO, Brasil; 5 - Professor da Área de Prótese e Odontogeriatria, Queen's University Belfast, Irlanda do Norte, Reino Unido.

\section{Resumo}

A técnica de cópia de próteses totais (PT), reconhecida como "copy denture", tem como principal benefício potencializar uma rápida adaptação ao novo par de próteses, uma vez que este irá possuir um formato já conhecido pelo paciente, mantendo tanto a posição dos dentes quanto sua dimensão vertical de oclusão. Apesar de haver relatos na literatura, esta técnica é pouco difundida e, muitas vezes, não é considerada como opção de tratamento em casos de pacientes idosos com maiores dificuldades potenciais de se adaptarem a novas próteses totais. Desta forma, o objetivo deste relato de caso é descrever o tratamento com próteses totais maxilar e mandibular utilizando-se a técnica de cópia. A primeira etapa consistiu na inclusão das próteses existentes em silicone extra duro laboratorial, seguido da confecção da base de prova com resina acrílica auto-polimerizável invertida no molde de silicone e, por último, o preenchimento da porção dentada do molde com cera 7 aquecida em fase líquida. A cópia em cera da prótese foi utilizada para averiguação em boca da relação maxilomandibular, do padrão oclusal, da dimensão vertical de oclusão (DVO) e também para realização da moldagem funcional com silicone de adição fluido. Os modelos de trabalho foram confeccionados e o conjunto foi montado em articulador semi ajustável para montagem dos dentes em cera. Em seguida, foi realizada a prova dos dentes em cera e verificação da oclusão, suporte de lábio e corredor bucal. Com a aprovação da paciente, as PTs seguiram para etapa de acrilização. Após instalação das próteses e finalização do tratamento reabilitador a paciente relatou uma mudança significativa na retenção e estabilidade de suas novas próteses, além de conforto e facilidade em se adaptar com o novo par de próteses. O caso foi acompanhado por 6 meses sem o surgimento de novas lesões em mucosa ou queixas da paciente. A técnica de cópia de próteses totais ("copy denture") demonstrou ser um método de confecção de PTs simplificado, com reduzidas etapas clínicas e laboratoriais, sendo uma possível alternativa à técnica convencional, especialmente para pacientes idosos que apresentam próteses totais sem alterações significativas.

PALAVRAS-CHAVE: Prótese total; Arcada Edêntula; Técnica de Moldagem Odontológica; Idoso; Relato de caso.

\section{AUTOR PARA CORRESPONDÊNCIA}

Faculdade de Odontologia, Universidade Federal de Goiás, Praça Universitária, s/n, Setor Universitário, 74605-220, Goiânia-G0, Brasil. Telefone: +55 (62) 32096052 / E-mail: claudio_leles@ufg.br 


\section{Introdução}

As próteses totais convencionais (PT) são o tratamento de escolha de muitos pacientes, devolvendo a eles não apenas aspectos funcionais, mas também psicológicos e sociais ${ }^{1}$.

Devido às remodelações do rebordo alveolar e desgastes dos dentes e estruturas de acrílico das próteses, a confecção de um novo par de próteses é aconselhada a ser realizada periodicamente, pelo menos a cada 10 anos $^{2}$. Contudo, apesar destas recomendações, é comum encontrar usuários de PT que utilizam o mesmo par de próteses por períodos excessivamente prolongados ${ }^{3,4}$, geralmente por falta de acesso ao tratamento odontológico ou receio em relação às dificuldades de se adaptarem às novas próteses.

A adaptação às novas próteses pode ser um desafio maior para pacientes idosos, principalmente quando são realizadas grandes mudanças oclusais e nas superfícies de suporte ${ }^{5}$. Desta forma, replicar os contornos e formato das próteses antigas de um paciente que as utiliza há vários anos, pode prevenir a necessidade e o desconforto de um recondicionamento da atividade muscular facial em decorrência à instalação de uma prótese com um novo formato ${ }^{6}$.

Os métodos de cópia de próteses totais (“copy denture"), também conhecidos como métodos de duplicação ou clonagem, são descritos em diversos protocolos na literatura ${ }^{7-9}$. Dentre estes, a técnica conhecida como "copy denture" tem como principal benefício potencializar uma rápida adaptação ao novo par de próteses ${ }^{10}$, uma vez que este irá possuir um formato já conhecido pelo paciente, mantendo tanto a posição dos dentes quanto sua dimensão vertical de oclusão ${ }^{10,11}$. Além disto, outros benefícios são citados, como o fornecimento de orientações à equipe técnica sobre a posição, estética e formato dos dentes; técnica de registro maxilo-mandibular mais simplificada; além da diminuição do número de visitas clínicas para confecção das próteses, redução das etapas laboratoriais e do tempo dos pacientes na cadeira odontológica ${ }^{12,13}$. 
Apesar destas vantagens, o cirurgião-dentista deve estar atento às indicações da técnica. Cada caso deve ser avaliado individualmente, por vezes o tempo de vida clínico da prótese não é o principal fator influenciador no emprego da técnica, mas sim o quanto suas estruturas de suporte e dentes se encontram preservados. A técnica de cópia é melhor empregada em casos que o paciente apresenta um certo grau de satisfação com as próteses antigas, apesar da solicitação e necessidade da confecção de novas ${ }^{9}$, devido a deficiências menores relativas à retenção, adaptação aos tecidos de suporte, ou estética insatisfatória por alterações dos dentes artificiais. Por outro lado, próteses com deterioração severa, como fraturas nas estruturas de suporte e/ou dentes, impedindo a reprodução da cópia das próteses; mau posicionamento dos dentes, com a dimensão vertical de oclusão (DVO) e a relação maxilo-mandibular excessivamente alteradas; ou com altos graus de insatisfação por parte do paciente, podem levar à contraindicação da técnica de cópia. A opção por copiar as próteses pré-existentes é inadequada nos casos em que as novas próteses requerem grandes alterações em relação às próteses existentes ${ }^{14}$.

Apesar de haver relatos na literatura ${ }^{6,10,11,13,14}$, esta técnica é pouco difundida e, muitas vezes, não é considerada como opção de tratamento em casos de pacientes idosos com maiores dificuldades potenciais de se adaptarem a novas próteses totais. Desta forma, o objetivo deste relato de caso é descrever o tratamento com próteses totais maxilar e mandibular utilizando-se a técnica de cópia.

\section{Relato de caso}

Uma paciente desdentada total, do gênero feminino, 69 anos de idade, cadastrada no Sistema de Regulação da Secretaria de Saúde de Goiânia, foi encaminhada à Faculdade de Odontologia da Universidade Federal de Goiás com indicação de confecção de um novo par de próteses totais. Durante anamnese, a paciente relatou que utilizava suas próteses há aproximadamente 40 
anos, após extração múltipla dos dentes remanescentes. A queixa principal era relacionada à necessidade percebida de troca das próteses por serem muito antigas. Apesar da aparência do par de próteses evidenciar seu tempo de vida clínica, quando questionada sobre seu conforto, estética e função, a paciente respondeu estar satisfeita em parte com todos estes parâmetros.

O exame inicial revelou uma condição estética moderadamente satisfatória (Figuras 1A e 1B). A DVO da paciente foi aferida com o auxílio de um compasso de Willis. As medidas do terço superior da face (canto externo do olho até a comissura labial) coincidiam com o terço inferior (região entre o ponto subnasal e o ponto gnático), após subtração do espaço funcional livre. As próteses antigas apesar de desgastadas garantiam à paciente uma DVO e articulação dentária adequadas. A PT maxilar apresentava pouca retenção, enquanto a PT mandibular apresentava baixa estabilidade e retenção. Além disto, ambas as próteses se apresentavam com manchas e com desgaste acentuado da base de resina acrílica e dentes (Figuras 1C e 1D). Os exames clínico e radiográfico apresentaram rebordos alveolares com altura preservada e sem alterações patológicas (Figura 1E e 1F).

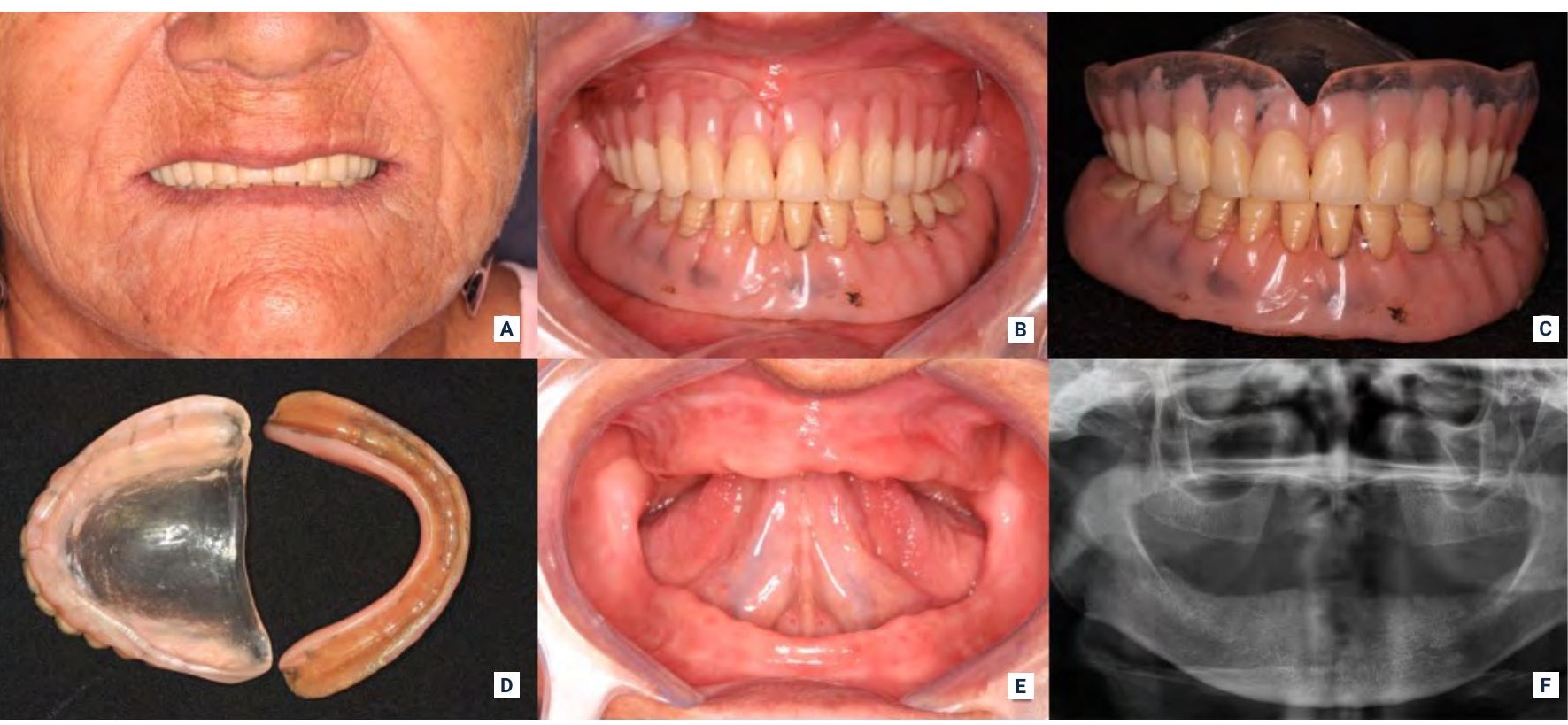

FIGURA 1 · (A-B) Vista frontal da condição inicial da paciente e das próteses; (C) Próteses atuais em posição; (D) Vista superior da porção interna das próteses; (E) Vista frontal das arcadas desdentadas e (F) radiografia panorâmica da paciente. 
O tratamento proposto à paciente foi a instalação de um novo par de próteses, mantendo-se a posição bucolingual dos dentes, dimensão vertical e padrão oclusal. A técnica de cópia das próteses existentes foi proposta e aceita pela paciente, tendo em vista a redução do número de visitas e o potencial de melhorar a adaptação funcional às novas próteses. Após leitura e assinatura dos Termos de Consentimento Livre e Esclarecido para a execução do tratamento proposto e para a divulgação do caso e das imagens, preservando a identidade da paciente, iniciaram-se as etapas para confecção do novo par de próteses. Por se tratar de procedimento coberto pelo convênio com o sistema público de saúde, não houve custos de tratamento para a paciente.

\section{Técnica}

Para a cópia das próteses foram selecionados dois pares de moldeiras metálicas para arcadas dentadas, compatíveis com o tamanho das próteses, e silicone extra duro laboratorial (Zetalabor; Zhermack, Badia Polesine, RO, Itália). Os mesmos passos foram seguidos separadamente para as próteses maxilar e mandibular.

O silicone foi manipulado e posicionado na moldeira e, em seguida, a prótese foi imergida no material de moldagem a partir da face oclusal, incorporando toda a parte externa da prótese até os limites de bordo. Após a cura do material, vincos em formato de "v" foram confeccionados, indo desde o limite do molde até o limite da moldeira, sem, no entanto, danificar o molde. Em seguida, toda a margem de silicone em volta do molde foi isolada com vaselina (Figura $2 \mathrm{~A}$ ).

A seguir, nova porção de material foi manipulada, preenchendo a parte interna da prótese e da moldeira, para que a segunda moldeira com silicone fosse sobreposta, copiando assim as superfícies internas da prótese (Figura $2 \mathrm{~B}$ ). Após a cura do material e separação das duas moldeiras, novos vincos foram confeccionados no segundo molde, em lugares coincidentes com os do primeiro, criando assim ductos de escoamento. 
O passo seguinte foi a confecção de bases de prova com resina acrílica incolor autopolimerizável (Vipiflash; VIPI, Pirassununga, SP, Brasil) sendo o material vertido somente sobre a cópia negativa do rebordo alveolar (Figura 2C). Após polimerização, os excessos foram removidos (Figura 2D) e a espessura das bases de provas verificadas para evitar futuras interferências durante a sobreposição dos moldes (Figura 2E).

Toda a extensão da cópia negativa dos dentes e palato foi então preenchida com cera 7 (Newwax 7; Technew, Rio de Janeiro, RJ, Brasil) aquecida e em fase líquida (Figura 2F), para que o segundo molde, com a base de prova em posição, fosse sobreposto, pressionando a cera líquida, fazendo com que os excessos escoassem pelos ductos previamente confeccionados. O conjunto de moldes de silicone/base de prova/cera líquida, foi mantido sob pressão manual até que toda a cera se resfriasse e possibilitasse a remoção da réplica da prótese em cera dos moldes (Figuras 3A e 3B).

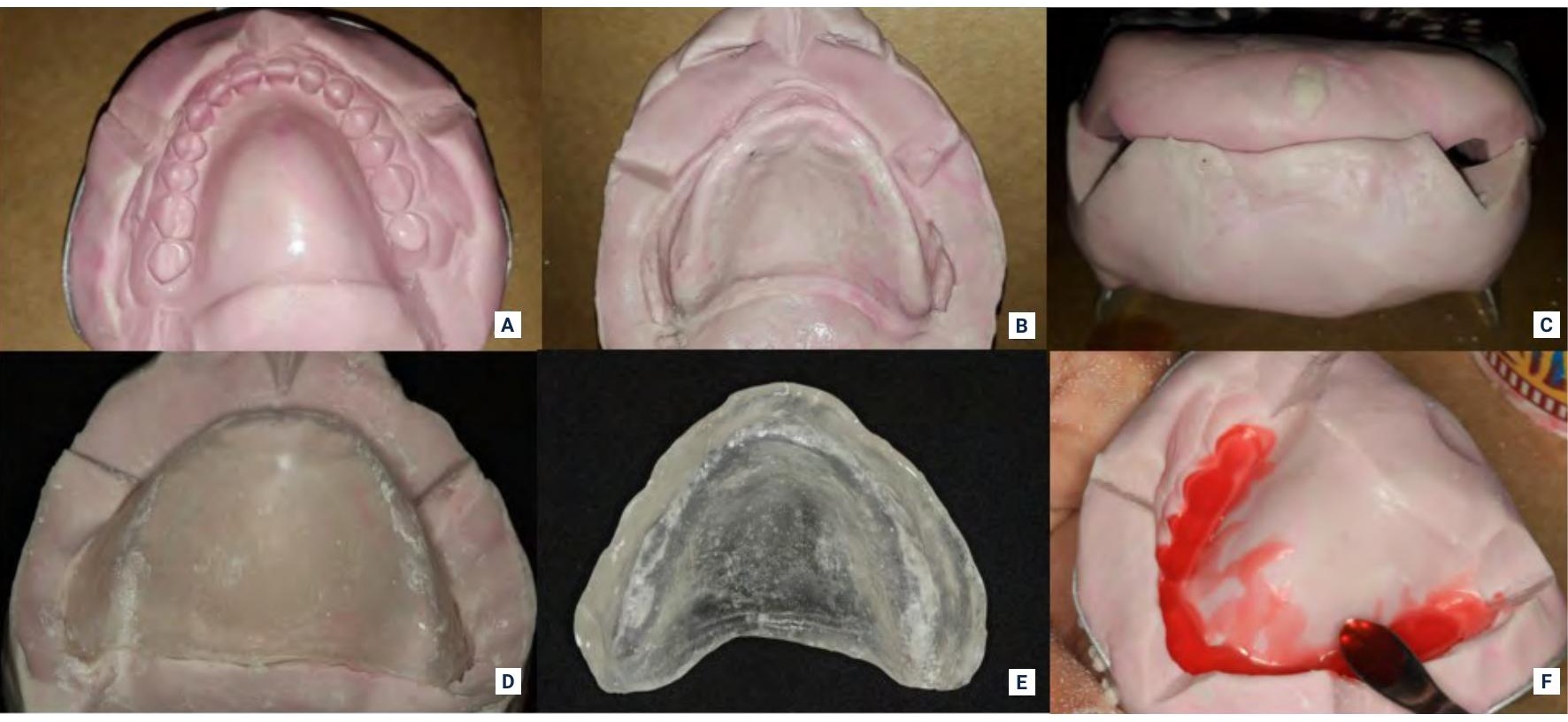

FIGURA 2 - (A) Vista superior do molde da prótese superior mostrando cópia negativa da face externa da prótese; (B): Vista superior do molde evidenciando a cópia das estruturas internas da prótese superior, além da presença dos vincos confeccionados em regiões coincidentes com os do primeiro molde; (C) Sobreposição dos moldes evidenciando a formação dos ductos de escoamento; (D) Base de prova em resina acrílica incolor logo após sua confecção e (E) Vista superior da base de prova, após refinamentos e aferição de sua espessura; (F) Cera 7 aquecida e em fase líquida sendo vertida no primeiro molde, recobrindo toda a porção dos dentes e palato. 
Em uma segunda sessão clínica, as réplicas das próteses superior e inferior foram provadas em boca, com o objetivo de avaliar a relação maxilomandibular, o padrão oclusal e a dimensão vertical de oclusão. Visto que no planejamento do caso foi prevista a manutenção dos parâmetros mencionados, apenas ajustes mínimos na extensão da base da prótese superior foram necessários com o acréscimo de uma pequena porção de guta percha em bastão (Godibar; Lysanda, São Paulo, SP, Brasil) aquecida, obedecendo os limites delimitados pelas fóveas palatinas, melhorando assim sua retenção.

As réplicas em cera foram utilizadas também para realização das moldagens funcionais. Para isto, as superfícies internas das bases de prova receberam uma porção de silicone de adição fluido (Express XT; 3M ESPE, St. Paul, MN, Estados Unidos da América), sendo inseridas novamente na boca da paciente, primeiramente a superior, seguida da inferior. A paciente foi orientada a movimentar a língua para as laterais e para fora, copiando assim o freio lingual e as inserções musculares desta região. Logo após o operador solicitou que a paciente ocluísse, sem grande força, sendo guiada à posição de relação cêntrica. Com o silicone ainda fluido, movimentos com lábios e mucosa jugal foram realizados, tracionando a musculatura e registrando agora as regiões de bridas, inserções musculares e freios labiais. A paciente então manteve as réplicas das próteses em oclusão até a reação final do material de moldagem (Figura 3C). Para que esta etapa fosse executada sem intercorrências, a paciente recebeu explicações prévias sobre as etapas da moldagem, os possíveis desconfortos gerados pela mesma, bem como sobre o posicionamento da língua e como deveria ocluir.

Logo após uma fina camada de cera aquecida foi colocada sobre o silicone do molde da arcada superior, em região de transição palato duro/palato mole, e levado novamente em posição realizando pressão contra o palato, a fim se realizar o selamento posterior (Figura 3D). Em seguida, as duas réplicas foram unidas 
na posição de relação cêntrica com uma camada de cera aquecida interposta entre os dentes. $O$ conjunto de réplicas unidas foi então removido, ainda em oclusão, aproveitando desta forma o registro maxilomandibular (Figura 3E). Os modelos de trabalho foram confeccionados com gesso especial (Figura 3F), e após a presa, o conjunto foi montado em articulador semi ajustável (4000-S; Bio-art, São Carlos, SP, Brasil) utilizando técnica simplificada sem o uso de arco facial (Figura 4A).

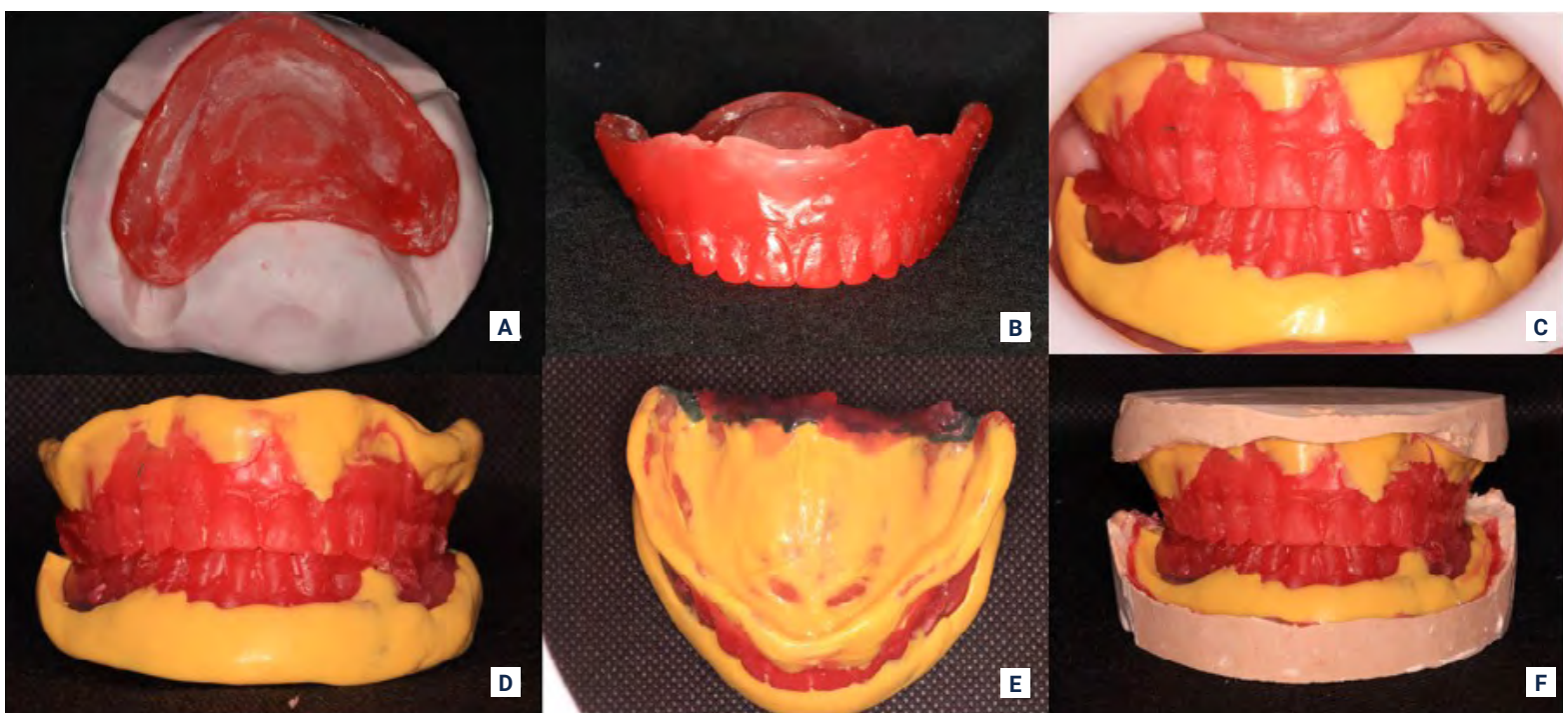

FIGURA 3 - (A) Imagem da réplica em cera da prótese superior após total resfriamento da cera e desencaixe dos moldes; (B) Réplica em cera da prótese superior após desinclusão dos moldes; (C) Réplicas sendo utilizadas para moldagem funcional com silicone de adição; (D-E) Vista do conjunto após remoção e selamento posterior realizado com cera; (F) Modelos de trabalho vazados com gesso especial.

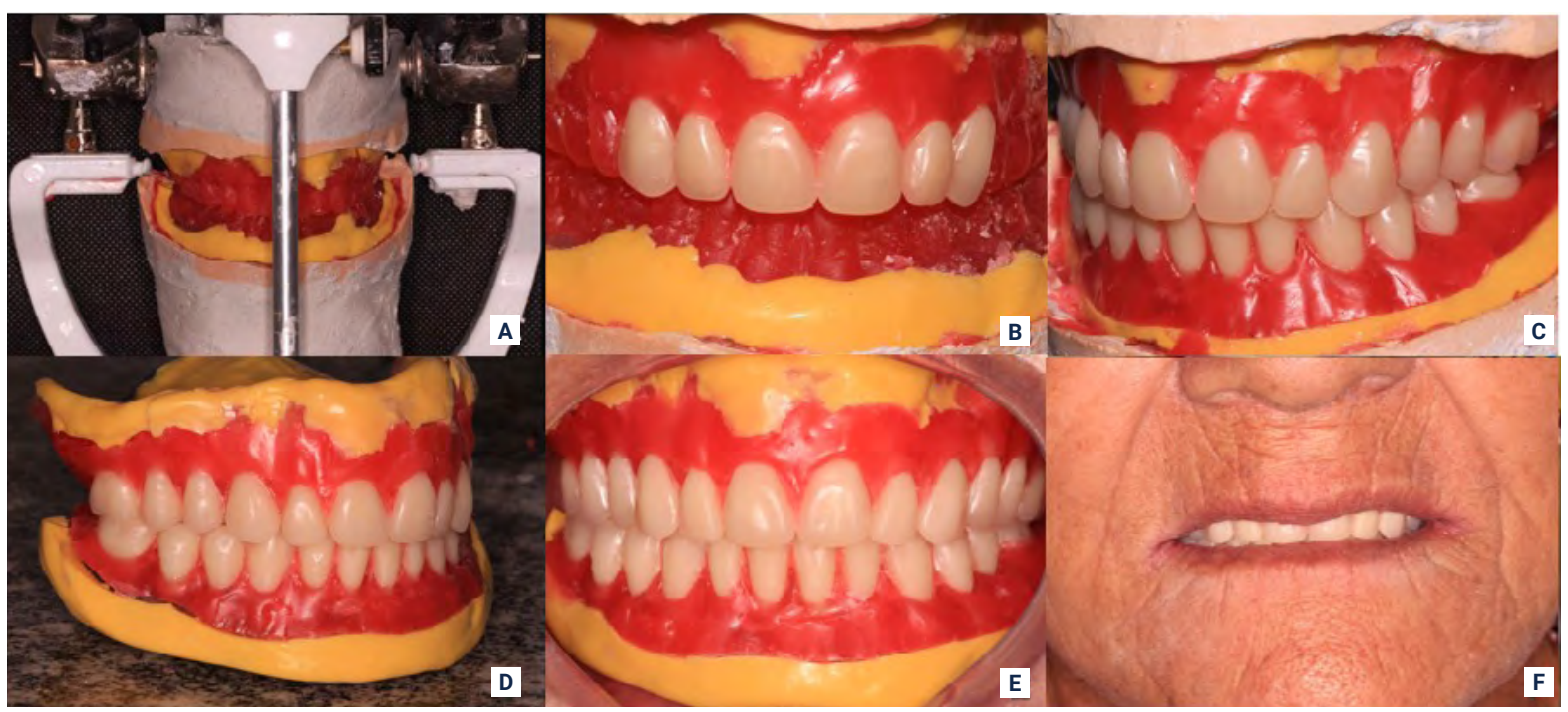

FIGURA 4 - (A) Modelos de trabalho montados em articulador semi ajustável; (B-C) Montagem dos dentes artificiais, utilizando os dentes de cera como referência; (D) Réplicas das próteses com montagem de dentes finalizada após remoção dos modelos; (E-F) Prova dos dentes. 
A seleção dos dentes levou em consideração as medidas obtidas a partir das réplicas dos dentes antigos em cera, escolhendo dentes com tamanho e formato semelhantes aos da prótese antiga. A cor dos dentes havia sido obtida na sessão anterior seguindo solicitações da própria paciente. Os dentes de cera foram recortados um a um, e substituídos pelos dentes artificiais (Trilux; VIPI, Pirassununga, SP, Brasil) (Figuras 4B e 4C), mantendo assim o padrão oclusal original como planejado (Figura 4D).

$\mathrm{Na}$ terceira consulta clínica foram avaliados a articulação dos dentes, o suporte labial e dos tecidos moles, havendo a necessidade apenas de ajustes mínimos, além de receber a aprovação da estética pela paciente (Figuras 4E e 4F). Após a etapa laboratorial de acrilização das próteses, seguindo procedimentos convencionais, a paciente foi requisitada para a consulta de instalação.

Durante a sessão de instalação, verificou-se o contorno periférico, a estabilidade das próteses sob pressão oclusal e digital, extensões posteriores, além aspectos fonéticos e de conforto (Figuras 5A e 5B). Os ajustes imediatos foram mínimos, e a consulta foi finalizada com instruções de higienização e manutenção da prótese. Todas as etapas clínicas e laboratoriais realizadas estão resumidas na Tabela 1 .
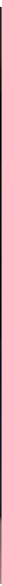

FIGURA 5 - (A) Imagem intrabucal das próteses finais instaladas; (B) Aspecto final do sorriso da paciente após instalação das novas próteses confeccionadas com a técnica de cópia. 
TABELA 1 • Etapas clínicas e laboratoriais para confecção das próteses pela técnica da cópia

\begin{tabular}{lll}
\hline ETAPAS & PROCEDIMENTOS & \\
\hline & CLÍ́NICOS & LABORATORIAIS \\
\hline I & Diagnóstico e planejamento do tratamento. & $\begin{array}{l}\text { Confecção dos moldes para duplicação; } \\
\text { Duplicação das próteses antigas e confecção de cópias } \\
\text { em cera. }\end{array}$ \\
\hline II & $\begin{array}{l}\text { Prova das réplicas das próteses em cera; } \\
\text { Moldagem funcional e registro interoclusal. }\end{array}$ & Montagem em articulador; \\
\hline Prova estética dos dentes & Mentagem dos dentes artificiais. \\
\hline vertical. & Acrilização, acabamento e polimento das próteses. \\
\hline & Instalação das novas próteses. & \\
\hline
\end{tabular}

Outros dois retornos foram necessários para realização de ajustes mínimos com desgastes para alívio em resina acrílica. Não foram observadas lesões extensas em mucosa que levassem ao impedimento do uso contínuo das novas próteses. A paciente relatou uma mudança significativa na retenção e estabilidade de suas novas próteses, além de conforto e facilidade em se adaptar com o novo par de próteses. O caso foi acompanhado por 6 meses sem o surgimento de novas lesões em mucosa ou queixas da paciente.

\section{Discussão}

O presente relato de caso objetivou descrever o tratamento com próteses totais superior e inferior confeccionadas pela técnica de cópia ("copy denture"). Essa técnica consiste na reprodução do formato e parâmetros gerais das estruturas de suporte das próteses, permitindo a realização de alterações mínimas de superfícies oclusais que, geralmente, se encontram desgastadas com o passar dos $\operatorname{anos}^{6}$. As novas próteses terão superfícies de acrílico e dentes íntegros, além de reproduzirem padrões oclusais já familiarizados pelos pacientes. 
Além disto, a técnica de cópia se aplica aos casos em que as próteses antigas apresentam características mínimas que permitam a reprodução de aspectos específicos das próteses, como o contorno das bases protéticas e posicionamento dos dentes. A seleção de casos deve ser bastante criteriosa e discutida com o paciente previamente, visto que grande parte das próteses que necessitam de substituição apresentam deficiências críticas que contraindicam a presente técnica, como falta de suporte labial, contorno dentário inadequado, alterações excessivas da dimensão vertical e da extensão da base da prótese.

No caso apresentado, optou-se por manter a dimensão vertical das próteses antigas da paciente, uma vez que estética, fonética e mastigação não estavam sendo prejudicadas por este parâmetro. Porém, é importante ressaltar que, em casos nos quais a DVO deve ser alterada, é possível realizar tais alterações durante a etapa da prova das réplicas em cera, acrescentando ou removendo material da superfície oclusal dos dentes inferiores em cera, até se atingir a dimensão desejada.

Outros autores trazem ainda técnicas de replicação das próteses com algumas modificações. Uma possibilidade é a confecção de toda a réplica em resina acrílica, até mesmo a região dos dentes $^{15,16}$. Esta alternativa, no entanto, dificulta a execução de alterações de padrões e dimensões oclusais e montagem de dentes, ao contrário da duplicação em cera que oferece uma maior liberdade de manipulação dos ajustes oclusais e montagem de dentes para a futura prótese.

Assim como a técnica da "copy denture", a técnica simplificada visa reduzir etapas clínicas e laboratoriais para a confecção de PT e, segundo a literatura, obtém resultados de satisfação com o tratamento reabilitador, similares aos obtidos pela técnica convencional ${ }^{17}$. Apesar de serem técnicas distintas, elas se assemelham pelo fato de que em ambas o cirurgião-dentista realizará apenas uma moldagem intrabucal, e também pela ausência do uso de arco facial para a montagem dos modelos em articulador. 
As duas são opções de técnicas alternativas à técnica convencional, não havendo, no conhecimento dos autores, estudos na literatura demonstrando vantagens sobre uma ou outra.

Como demonstrado no relato de caso, a técnica da "copy denture" é realizada utilizando apenas equipamentos ou materiais que estão na rotina clínica do cirurgião-dentista, sendo possível a realização de todas as etapas em seu consultório até a prova dos dentes em cera, deixando apenas a acrilização como etapa laboratorial. Contudo, visando um melhor aproveitamento do tempo clínico do cirurgião é desejável a coparticipação de um técnico de prótese dentária para a realização de etapas como a confecção da prótese em cera após a moldagem, montagem dos dentes em cera e acrilização.

A tecnologia "computer-aided-design computer-aided-manufacturing” (CAD-CAM), traz ainda uma amplificação nas possibilidades de realização das técnicas de réplica de próteses. Davda et al. ${ }^{10}$ (2017), traz em seu estudo laboratorial réplicas de PT confeccionadas com a técnica "convencional” comparadas com réplicas obtidas por meio da tecnologia CAD-CAM e impressoras $3 \mathrm{D}$. Os resultados mostraram que a segunda opção apresenta cópias com dimensões mais precisas do que a técnica tida como “convencional”. Apesar do crescente uso destas tecnologias no mercado odontológico, elas ainda oneram o valor final do tratamento, uma vez que seus materiais e equipamentos ainda não são economicamente viáveis.

A redução no valor final das próteses, por consequência do menor número de consultas necessárias para finalização do tratamento reabilitador, é levantada como um dos benefícios da técnica de cópia convencional ${ }^{18}$. Outro aspecto já mencionado é a manutenção de padrões oclusais e do formato das próteses antigas. Estes fatores podem ajudar na adaptação de pacientes idosos ao novo par de próteses, uma vez que estes pacientes apresentam dificuldades em se adaptar a um novo formato de prótese, devido à necessidade de recondicionarem suas 
atividades musculares quando estas são instaladas ${ }^{19}$. Isto parece ser ainda mais enfatizado em pacientes que utilizam as próteses antigas há vários anos ${ }^{6}$, como foi o caso descrito neste trabalho, em que a paciente utilizava o mesmo par de próteses há 40 anos, o que poderia ser um fator limitador na adaptação às novas próteses. Contudo, ao final do tratamento, quando questionada sobre conforto e adaptação, a paciente demonstrou grande satisfação com as próteses recebidas.

Apesar dos benefícios levantados sobre as técnicas de réplica de PTs quando comparados com as técnicas de confecção convencionais, há ainda autores que refutam estas informações. Scott et al. ${ }^{20}$ (2006), desenvolveram um estudo clínico comparando parâmetros de satisfação dos pacientes reabilitados com próteses confeccionadas com técnicas convencionais e técnicas de duplicação das próteses pré-existentes. Os autores encontraram uma melhora apenas no quesito "constrangimento" no grupo reabilitado com a técnica de duplicação da prótese. Contudo, há algumas ressalvas no estudo. Além de o próprio autor ressaltar o pequeno número de participantes, as próteses foram confeccionadas por alunos de graduação que estavam iniciando suas atividades clínicas na área de reabilitação. Por isto, sugere-se a realização de ensaios clínicos randomizados que comparem pacientes tratados com próteses confeccionadas pelos métodos convencional e de cópia.

Finalmente, apesar das poucas evidências sobre a técnica apresentada, o presente caso apresentou resultados plenamente satisfatórios, com a paciente mostrando grande satisfação com as próteses recebidas, além de relatar facilidade em se adaptar às mesmas. Desta forma, este trabalho sugere que a técnica de cópia pode ser uma alternativa viável, mais simplificada e de menor custo em relação à técnica convencional de confecção de próteses totais, sendo melhor indicada para pacientes que necessitam de condições mais favoráveis para superarem o período inicial de adaptação às novas próteses. Contudo, mais estudos clínicos atuais são necessários para que se comprovem maiores benefícios sobre essa técnica. 


\section{Conclusão}

A técnica de cópia de próteses totais (“copy denture”) demonstrou ser um método de confecção de PTs simplificado, com reduzidas etapas clínicas e laboratoriais, sendo uma possível alternativa à técnica convencional, especialmente para pacientes idosos que apresentam próteses totais sem alterações significativas.

\section{Referências}

1 - Papadaki E, Anastassiadou V. Elderly complete denture weares: a social approach to tooth loss. Gerontology. 2012; 29(2): 721-727.

2- Taylor M, Massod M, Mnatzaganian G. Longevity of complete dentures: A systematic review and meta-analysis. The Journal of Prosthetic Dentistry. 2020 [Epub ahead of print].

3 - Coelho CM, Sousa YT, Daré AM. Denture-related oral mucosal lesions in a Brazilian school of dentistry. J Oral Rehabil. 2004; 31(2): 135-139.

4 - Peracini A, Andrade IM, Paranhos HFO, Silva CHL, Souza RF. Behaviours and Hygiene Habits of Complete Denture Wearers. Braz Dent J. 2010; 2(3):247-252.

5 - MacEntee MI. The effects of aging on the edentulous state. In: Hobrink J, Zarb GA, Bolender CL, Eckert S, Jacob R, Fenton A, Mericske-Stern R. Prosthodontic treatment for edentulous patients: complete dentures and implant-supported protheses. St. Louis: Mosby; 2004. p. 03-05.

6 - McCord JF, Hannah VE, Cameron D, Watson D, Donaldson AC. An update on the replica denture technique. Dent Update. 2010; 37(4): 230-232, 235.

7 - Sandra L, McCarthy SL. Fabrication of a duplicate denture from an existing complete denture. J Prosthod. 1995; 4(1): 54-57.

8 - Lindquist TJ, Narhi TO, Ettinger RL. Denture duplication technique with alternative materials. J Prosthet Dent. 1997; 77(1): 97-98.

9 - Owen CP. New dentures from old: A duplication method using "Appropriatech". JCDA. 2006; 72(5): 393-7.

10 - Davda K, Osnes C, Dillon S, Wu J, Hyde P, Keeling A. An investigation into the trueness and precision of copy denture templates produced by rapid prototyping and conventional means. Eur J Prosthodont Restor Dent. 2017; 25(4): 186-192.

11 - Soo S, Cheng AC. Complete denture copy technique: a practical application. Singapore Dent J. 2014; 35: 65-70.

12 - Paterson AJ. Removable Prosthodontics. In: Churchill's Pocket Book of ClinicalDentistry. 2nd ed. London: Elsevier; 2002. p. 201-260. 
13 - Habib SR, Vohra FA. Replacing Existing Dentures by CopyDenture Technique for Geriatric Patients: A Case Report. JPDA. 2013; 22(4): 265-270.

14 - Jablonski RY, Patel J, Morrow LA. Complete dentures: an update on clinical assessment and management: part 2. Br Dent J. 2018; 225(10): 933-939.

15 - Diniz AC, Ribeiro JA, Tôrres ACSP, Carreiro AFP. Duplicação rápida de prótese total: passo-a-passo. Revista Ciência Plural. 2015; 1(3): 85-92.

16 - Shor A, Shor K, Goto Y. An alternative technique for the fabrication of removable complete dentures. Pract Proced Aesthet Dent. 2007; 19(6): 337-344.

17 - Nuñez MCO, Silva DC, Barcelos BA, Leles CR. Patient satisfaction and oral health-related quality of life after treatment with traditional and simplified protocols for complete denture construction. Gerontology. 2015; 32(4): 247-53.

18 - Clark RKF, Radford DR, Fenlon MR. The future of teaching of complete denture construction to undergraduates in the $\mathrm{UK}$ : is a replacement denture technique the answer? British Dental Journal. 2004; 196(9): 571-575.

19 - Brill N, Tryde G, Schübeler S. The role of learning in denture retention. J Pros Dent. 1960; 10(3): 468-475.

20 - Scott BJJ, Forgie AH, Davis DM. A study to compare the oral health impact profile and satisfaction before and after having replacement complete dentures constructed by either the copy or the conventional technique. Gerontology. 2006; 23(2): 79-86. 


\title{
Copy denture technique for elderly patients: a case report
}

\begin{abstract}
The "copy denture" technique has been proposed as an alternative approach for complete denture fabrication, aiming to increase the potential of patient adaptation to the new dentures, due to the partial reproduction of the old dentures that the patient is accustomed using, duplicating both the position of the teeth and the vertical dimension of occlusion. Although there are previous reports in the literature, this technique is not widespread and is often not considered as a treatment option in cases of elderly patients with great difficulties in adapting to new dentures. Thus, the objective of this case report is to describe the treatment with maxillary and mandibular complete dentures using the copy denture technique, including the clinical and laboratory steps. After the conclusion of the treatment, the patient reported a significant improvement in the retention and stability of the new dentures, greater comfort, and easily accustomed in a short-term period. The copy denture technique is a simplified technique for the fabrication of complete dentures, with reduced clinical and laboratory steps, and may promote better treatment outcomes for elderly patients.
\end{abstract}

KEYWORDS: Complete Denture; Edentulous Jaw; Dental Impression Technique; Aged; Case Report.

\section{Como citar este artigo}

Paula MS, Magalhães-Neta A, Oliveira TMC, Leles CR, McKenna G. Técnica de cópia de próteses totais para pacientes idosos: relato de caso. Rev Odontol Bras Central 2021; 30(89): 4-19. DOI: 